As the field reflects on Riggs et al., I beg its indulgence for this brief intrusion, and for the reminder: a quarter of a century later, the cuprates still retain their mystique. And their preeminence at the frontier of condensed-matter physics.

Zlatko Tesanovic is at the Institute for Quantum Matter and the Henry A. Rowland Department of
Physics and Astronomy, Johns Hopkins University, Baltimore, Maryland 21218, USA.

e-mail:zbt@pha.jhu.edu

\section{References}

1. Doiron-Leyraud, N. et al. Nature 447, 565-568 (2007).

2. Sebastian, S. E. et al. Nature 454, 200-203 (2008).

3. Chakravarty, S. Rep. Prog. Phys. 74, 022501 (2011).

4. Scalapino, D. J., Loh, E. \& Hirsch, J. E. Phys. Rev. B 34, 8190-8192 (1986)
5. Anderson, P. W. Science 22, 1705-1707 (2007).

6. Metlitski, M. A. \& Sachdev, S. Phys, Rev, B 82, 075128 (2010)

Berg, E., Fradkin, E. \& Kivelson, S. A. Phys. Rev. B 79, 064515 (2009).

8. Millis, A. J. \& Norman, M. R. Phys. Rev. B 76, 220503(R) (2007)

9. Tesanovic, Z. Nature Phys. 4, 408-414 (2008)

10. Li, L. et al. Nature Phys. 3, 311-314 (2007).

11. Chatterjee, U. et al. Nature Phys. 6, 99-103 (2010).

12. Riggs, S. C. et al. Nature Phys. 7, 332-335 (2011).

\title{
SIMON VAN DER MEER
}

\section{Stochastic success}

Simon van der Meer, who died on 4 March 2011 at the age of 85, followed a path to Stockholm that was perhaps less orthodox than most. A native of The Hague in the Netherlands, he finished his high-school science studies in 1943 but was unable to progress to university for two years, owing to the closure of the Dutch universities under the German occupation. At the end of the war, he entered the University of Technology at Delft to study 'technical physics', a subject more akin to engineering. "I have often felt regrets", he wrote at the time of the award of his Nobel Prize in Physics in 1984, "at not having had the intensive physics training that many of my colleagues enjoyed."

Nevertheless, van der Meer was building a successful career, first at the Philips Research Laboratory in Eindhoven and then, from 1956, at a recently founded laboratory in Geneva, Switzerland CERN. His work was technical in nature, mostly concerned with magnets and power supplies for CERN's $28 \mathrm{GeV}$ synchrotron, but interaction with accelerator and experimental physicists at the laboratory led him to develop an interest in particles, and how to handle them in an accelerator.

By the early 1960s, he had devised the 'horn' that, by enabling the focusing of neutrinos into beams of sufficient intensity, became a crucial component of most neutrino experiments - particularly those present-day experiments in which neutrino beams are fired through the Earth over distances of hundreds of kilometres to explore the tendency of these particles to oscillate from one type of neutrino to another.

In 1968, in a meeting at CERN, van der Meer first raised his notion of 'stochastic

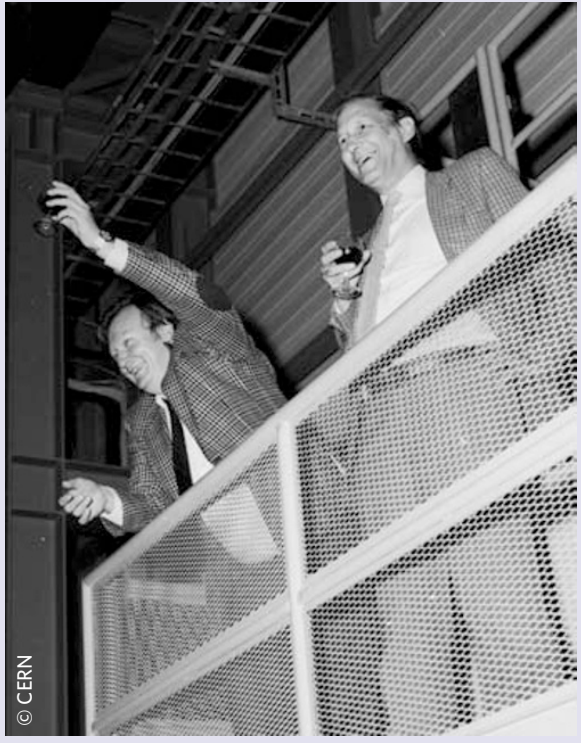

The problem lay in accumulating a sufficient number of particles, nestled tightly together in the accelerator beams, to achieve a high-enough rate of collisions to find the bosons. Van der Meer's stochastic cooling was the solution, and he proved its efficacy in the Initial Cooling Experiment before it was deployed in the SPS.

Stochastic cooling works by reducing the spread in the momentum of the particles within each of the 'bunches' that make up an accelerator beam. As the bunches circulate in the accelerator electrical signals are picked up from individual particles within a bunch and used to activate an electrical 'kicker' that pushes the particle's momentum back into line. According to a statement from CERN Director General Rolf-Dieter Heuer and Director of Accelerators Steve Myers, stochastic cooling is "deceptively simple at first sight, but to anyone who truly understands accelerators it was nothing less than a stroke of genius."

Van der Meer's genius coupled with Rubbia's drive was a winning formula. In 1981 the SPS began generating protonantiproton collisions, using stochastic cooling; in 1983, the existence of the $W$ and $Z$ bosons was confirmed; and in 1984, the Nobel Prize was shared, and celebrated, by van der Meer and Rubbia (pictured right and left, respectively). Despite the Nobel, modesty always prevailed for van der Meer: "If I have at times been able to make original contributions in the accelerator field, I cannot help feeling that to a certain extent my slightly amateur approach in physics, combined with much practical experience, was an asset."

ALISON WRIGHT 